\title{
Validity of Single Leg Hop Test in Comparison of Lysholm Score after ACL Reconstruction through Anteromedial Portal Technique by Using Hamstring Tendon
}

\author{
Gupta Anuj ${ }^{1}$, Ganvir A ${ }^{2}$, M.S. , Pathak A ${ }^{3}$, M.S., Gaur Sanjiv ${ }^{4}$, M.S., D.N.B., M.Ch. \\ 1,2,3,4 (Department of Orthopaedics, Gandhi Medical College, Bhopal, MP, India)
}

\begin{abstract}
:
Introduction: The anterior cruciate ligament (ACL) is the most frequently injured knee ligament, accounting for about 50\% of all knee ligament injuries The principle function of the ACL is to limit anterior translation of the tibia on the femur. Patients with a lesion of the ACL are characterized by difficulty with athletic performance and giving way symptoms in daily life activities. The purpose of this study is to investigate the short-term functional and clinical outcomes after anatomic ACL reconstruction by single bundle technique with the help of Lysholm scoring system and to assess the validity of the functional test (one leg hop test.

Materials \& methods: This cohort study included 25 patients (2 females and 23 males), mean age 28.4(1849yrs), with unilateral ACL tear who underwent ACL reconstruction by anteromedial portal technique using 4 strand hamstring graft. Follow up was done in 24 patients (1 patient lost due to infection and graft removal) at a median time of 7.5 months (6 to 11 months) who received standard outpatient and home based rehabilitation and analysis of Lysholm score, single leg hop test and patients' satisfaction with outcome (VAS) was done.

Results: Pre-operative lysholm score, instability subscore, pain subscore and single hop \% was 49.12, 8.95, 10.20 and $21.96 \%$ respectively. At the follow up Lysholm score was 89.96+-7.9(0-100), the lysholm instability subscore was 23.33+-3.18 (0 -25), the lysholm pain subscore was 20.42+-3.58 (0 -25), single leg hop test was $69.09+-12.08 \%$ of the normal limb and patients satisfaction score by VAS was 7.6 (0 -10). Excellent results were found in $37.5 \%$ patients, good results in $54.16 \%$ and poor in $8.3 \%$ patients. The score and hop test $\%$ as compared to pre-operative values were found to be highly significant with $p$ value $<0.001$. The highest correlation coefficient was found between lysholm score and single leg hop test $(p=0.64)$ proving its validity, and between score and patients' satisfaction $(p=0.75)$.

Conclusion: We conclude that the anteromedial portal technique and anatomic placement of grafts is an effective surgical technique for treating ACL deficient knees. It restores stability with excellent clinical and functional results. Also single leg hop test is a useful and reliable functional test for evaluating post-operative outcome after an ACL reconstruction.
\end{abstract}

Keywords: ACL, ACL Reconstruction, hamstring graft, Lysholm score, satisfaction, single leg hop

\section{Introduction}

The anterior cruciate ligament (ACL) is the most frequently injured knee ligament, accounting for about $50 \%$ of all knee ligament injuries and the majority of injuries occur during sports activities which are increasing in incidence as more persons are involving in sports. ${ }^{[1,2]}$ Patients with a lesion of the ACL are characterized by difficulty with athletic performance and giving way symptoms in daily life activities. ${ }^{[3]}$ The principle function of the ACL is to limit anterior translation of the tibia on the femur. Non-surgical management of this injury may be appropriate in certain instances, however, it is widely accepted that for symptomatic instability an ACL reconstruction is critical for the prevention of secondary injury and long-term morbidity. ${ }^{[3-6]}$ The optimal surgical technique for ACL reconstruction remains a topic of controversy. The transtibial (TT) technique where the femoral tunnel is drilled through the tibial tunnel is the most traditional and popular technique ${ }^{[7]}$ with its main advantage being less surgical time. Recent studies found that the TT technique results in graft placement in a more vertical position and the anteromedial (AM) technique where the femoral tunnel is drilled through an arthroscopic portal with the knee in maximum flexion allows the surgeon more flexibility to place the graft in an oblique position. ${ }^{[8,9]}$

Current thinking suggests that reconstructing the ACL with an anatomically placed graft has the potential to maximize outcomes. Some studies have demonstrated superior outcomes and improved rotational stability with double-bundle reconstruction compared with single-bundle reconstructions. ${ }^{[10,11]}$ However, as of late, there have been reports of no difference in outcome measures between the two techniques particularly when the reconstruction is "anatomic". ${ }^{[12,13]}$ Patient satisfaction after "anatomic" Reconstruction of the anterior cruciate ligament has not been well studied. ${ }^{[14]}$ 
Over the past 20 years, focus on patient-reported outcomes rather than clinician-based measures has increased due to the superior validity of the former. ${ }^{[14,15]}$ The Lysholm score, developed in $1982^{[16]}$ is one of the most extensively used outcome scales in both clinical and research fields and has been proven valid and reliable in patients with ACL injury. ${ }^{[16,17]}$ The priority objective of this study is to evaluate functional and clinical outcomes after anatomic ACL reconstruction by single bundle technique with the help of Lysholm scoring system and to assess the validity of the functional test (one leg hop test) and patients' satisfaction.

\section{Materials And Methods}

Twenty five patients of anterior cruciate ligament deficient symptomatic knee were included in the present study which was carried out at department of Orthopaedics and Traumatology, Gandhi Medical College and Hamidia Hospital, Bhopal from October 2013 to September 2015. Out of a total of 25 patients who underwent ACL reconstruction using quadrupled hamstring tendon 24 were followed in OPD. One patient was lost in follow up and was excluded from the study. Majority of the patients were males i.e. 22 patients $(91.67 \%)$ and 2 were females $(8.3 \%)$ with mean age of 28.4 years. Right knee was involved in 13 patients $(54.16 \%)$ and left in 11 patients $(45.84 \%)$. The majority of injuries occurred during road traffic accidents $(n=16) ; 4$ patients were injured during sports activities and 4 during work. All the patients were assessed clinically and confirmed by MRI. The lysholm score and single leg hop test were assessed both pre and post operatively. This test was performed by jumping and landing on the same foot with the hands behind the back. Three attempts were to be made for each leg and the longest hop was recorded. A quotient (\%) between the injured and non-injured leg was calculated. Also patient satisfaction was assessed by using visual analogue scale (VAS) with a paper questionnaire asking "how satisfied are you with your outcome?" and scored ranging from 1 to 10 ranging from "very unsatisfied" to "very satisfied" respectively. Median time of follow up was 7.5 months (6 -11 months) with a minimum follow up of 6 months. Patients in whom lysholm score was more than 92 points were considered as excellent, scores between 91 and 76 points were rated as good and scores less than 76 points were rated as fair/poor.

\subsection{Inclusion and exclusion criteria}

Patients were included with ACL tear with or without meniscal injury, aged 17 to 50 years, who were willing for consensual follow up of minimum of 6 months and who were willing for OPD and home based rehabilitation. Patients excluded were those having other ligaments injury in the same and contralateral knee, with intra-articular cartilage damage and with osteoarthritic lesions in the knee joint.

\subsection{Surgical procedure}

A diagnostic arthroscopy was performed and a full assessment of all intra-articular structures was performed. Those having meniscal tears were treated surgically by partial menisectomy prior to reconstruction and the ACL stump was shaved off next.The femoral insertion site is at the 2 o'clock position on a left knee and the 10-o'clock position on a right knee. The knee was hyper-flexed to 120 degrees for better visualization and passing the guide wire which was then drilled and came out from lateral aspect of thigh piercing both the cortices. The elbow ACL tibial guide was set to an angle of $55^{\circ}$ and placed in the center of the remaining tibial stump of the native ACL through the medial portal. The free edge of the anterior horn of the lateral meniscus, center of medial and lateral tibial spines and point $5 \mathrm{~mm}$ to $7 \mathrm{~mm}$ anterior to PCL was often used as the aimer point. Femoral side was fixed with either interference screw or endobutton and tensioning of the graft was achieved by cycling the knee around 20 times from 0 to $90^{\circ}$. Tibial side was fixed with interference screws in all cases while tensioning the graft and giving posterior drawer at the same time. (Fig. 1.)



Figure 1. ACL reconstruction intraoperative image 


\subsection{Rehabilitation protocol}

The postoperative rehabilitation was standardized for all patients. Long knee brace was used in all patients. The brace is initially locked in full extension for a week, however, intermittent quad sets, straight leg raises, heel slides and calf pumps are encouraged. A continuous passive motion machine was used for the first 2 weeks. Weight bearing was started usually on day 2. Patients were discharged from the hospital on day 3. Postoperative full extension was allowed with a limitation of flexion to $90^{\circ}$ for 4 weeks. Patients attended outpatient rehabilitation and physical therapy for a period of 6 to 12 weeks. Rehabilitation included stationary bicycle after 4 weeks and jogging 3 months postoperatively. Closed-chain exercises were encouraged immediately postoperatively and open-chain exercises were allowed at 3 months postoperatively. Return to specific sports activity was allowed at 6 months after surgery.

\subsection{Statistical analysis:}

Statistical analysis was performed using the software SPSS version 20.0 for Windows. Paired t-test and analysis of variance was used for statistical significance. For power analysis the alpha error was fixed at $5 \%$ (confidence interval 95\%). The level of significance was $\mathrm{p}<0.05$.

\subsection{Surgery interval}

\section{Observations \& Results}

Mean time between trauma and surgery was 11.03 months. 11 patients were operated within the first 3 months after trauma, 7 patients within the first year and 6 patients after 1 year of initial trauma.

\subsection{Complications}

There were no intra- or postoperative complications during the in-patient period. Only one out of 24 patients showed signs of superficial infection over the graft removal site and portal sites but improved after oral antibiotics for 2 weeks.

One patient had persistent pain at follow up having score of 4 on visual analogue scale. The one patient which was lost in follow up had deep infection for which graft removal was done and hence excluded from the study.

\subsection{Score results}

The mean Lysholm score increased from pre-operative value of $49.12 \pm 14.1$ to $89.96 \pm 7.9$ at final follow up, the mean instability sub score increased from $8.96 \pm 4.6$ to $23 \pm 3.18$ and mean pain sub score also increased from $10.20 \pm 5.4$ to $20.42 \pm 3.5$. All these results were found to be statistically significant $(p<0.05)$ (Fig. 2). Nine patients showed excellent results with score $>92$, thirteen patients showed good results with score between 76 and 92 and two patients showed poor results with score <76. (Fig. 3.)



Figure 2. Mean value summary of lysholm score 


\section{RESULTS}



Figure 3. Graphical presentation of final result

\subsection{Single leg hop test}

Mean value of Single leg hop test (Fig. 4) improved from $21.96 \pm 10$ preoperatively to $69.1 \pm 12.8$ at the time of follow up and is found to be significant $(p<0.05)$

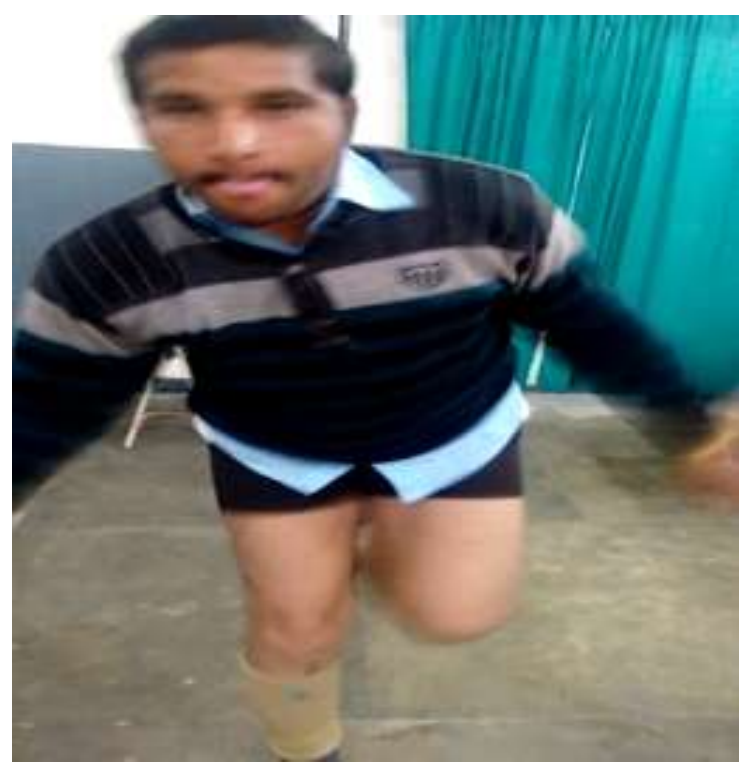

Figure 4. Single Leg Hop Test

\subsection{Analysis of subgroups}

Mean Lysholm score at follow up was found to be positively co-related with mean patients' satisfaction having Pearson co-relation coefficient of $0.76(p<0.05)$. Also, a positive co-relation was found between mean Lysholm score and mean single leg hop results with coefficient $0.64(p<0.05)$ (Table 1.)

Table 1.

\begin{tabular}{|c|c|c|c|c|c|}
\hline & Mean & Std. Deviation & $n$ & $\begin{array}{l}\text { Pearson Co } \\
\text { relation }\end{array}$ & p value \\
\hline SATISFACTION & 7.667 & 1.3962 & 24 & 0.759 & 0.000 \\
\hline HOP\% & 69.09 & 12.081 & 24 & 0.64 & 0.000 \\
\hline
\end{tabular}




\subsection{Final subjective results}

On a visual analogue scale from 0 to 10 average score for patient satisfaction is 7.6. Thirteen patients were very satisfied (score 8-10) with their results, nine patients were satisfied (score 6-7) and two patients were unsatisfied (score $\leq 5$ ). Similarly on a scale of 0 to 10 ranging from no pain to worst pain mean value of pain improved from 6.3 preoperatively to 1.5 at follow up.

\section{Discussion}

P. Saravanan, Dobson Dominic ${ }^{[18]}$ showed most of the surgeries within 3 months of injury in their study. The mean injury to surgery time in our study was 11.03 months with majority of surgeries (11 patients, $45.83 \%$ ) done within 3 months of injury.

P. Saravanan, Dobson Dominic ${ }^{[18]}$ showed common mode of injury as Road Traffic Accident (RTA). M.N. Tahmasebi, M. Shahrezaee, et al ${ }^{[19]}$ showed most common cause of tear to be during playing soccer. Matthias Buchner, et al ${ }^{[20]}$, also showed football to be the major cause of injury. The majority of injuries occurred in our study were during road traffic accidents $(n=16 ; 66.67 \%)$. In Indian Scenario most of the people go outside their homes for job through their own vehicle during congested traffic.

The surgical technique used in our study is of single bundle reconstruction using the anteromedial portal in which the femoral tunnel was made independently of the tibial tunnel placing the graft more anatomical and giving better stability and clinical results which has been proved by various studies like Christopher D. Harner et al ${ }^{[21]}$. Steiner Mark et al ${ }^{[22],}$ Eduard Alentorn Geli, Gonzalo Samitier et al ${ }^{[23]}$.

Matthias Buchner, et al ${ }^{[20]}$ showed complications like re surgery in $13 \%$ of patients and re rupture at follow up in $7 \%$ of patients. On the other hand Andrea Ferretti et al ${ }^{[24]}$ showed no complications at all. Complication in our study is only $8.33 \%$ with one patient showing signs of infection and one patient having persistent pain at 6 months of follow up.

Matthias Buchner, et $\mathrm{al}^{[20]}$ showed the mean Lysholm score at time of follow-up to be $83.64( \pm 15.9)$ in which twenty patients (29\%) were rated as excellent, thirty nine patients $(56 \%)$ as good and ten patients $(15 \%)$ as fair or poor which is very similar to our study. H. E. Bourke, D. J. Gordon et al ${ }^{[25]}$ showed the mean Lysholm knee score at 15 years to be 93; a total of 143 patients (94\%) had a good or excellent Lysholm score at one year and $134(88 \%)$ had good or excellent scores at 15 years. Andrea Ferretti et al ${ }^{[24]}$ showed improvement of Lysholm score from 57 preoperatively to 96 at follow up $(P<0.05)$. In our study a significant improvement is shown in the mean Lysholm score which increased from $49.12 \pm 14.1$ to $89.96 \pm 7.9$ at final follow up ( $<<0.05$ ). Susan L. Keays, et al ${ }^{(26)}$ showed single leg hop index improved from 0.79 to 0.99 after using semitendinosus grafts. Vernon J. Cooley, et al ${ }^{[27]}$ showed that all patients were within $10 \%$ of the normal leg for single leg hop test. Our study showed significant improvement in single leg hop results from $21.96 \% \pm 10$ preoperatively to $69.1 \% \pm 12.8$ at follow up $(p<0.05)$. These studies support results in the current study proving ACL reconstruction by the used method to be functionally sound and reliable. The reliability of single leg hop test have been proved by many studies ${ }^{[28,29,30]}$ but to prove its validity comparison with other outcome measures is justified ${ }^{(31)}$. In this study the one-leg-hop test results were $\approx 70 \%$ of the non-injured knee, which is confirmed as a normal value by various studies and is positively co-related with the Lysholm score $(\rho=+0.64)$ proving validity of this test.

Mohsen Mardani, et al ${ }^{[32]}$ in their study had mean satisfaction score of $9.72 \pm 0.5$ with high patient satisfaction. Matthias Buchner, et al ${ }^{[20]}$ showed the score of $8.14 \pm 2.25$ with $73 \%$ very satisfied, $23 \%$ satisfied and $4 \%$ unsatisfied. Eduard Alentorn-Geli, et al ${ }^{[23]}$ also resulted in high patient satisfaction with mean score of 9.1. In our study the mean VAS score for patient satisfaction is 7.6. Thirteen patients were very satisfied with their results, nine patients were satisfied and two patients were unsatisfied. Present study has high patient satisfaction after final outcome supported by above studies.

The mean time of follow up in different studies are shown in Table 2.

Table 2.

\begin{tabular}{|c|c|}
\hline STUDY & MEAN FOLLOW UP TIME \\
\hline Matthias Buchner, et al ${ }^{[20]}$ & 6 years and 4 months \\
\hline P. Saravanan, Dobson Dominic ${ }^{[18]}$ & 2 years \\
\hline Vernon J. Cooley, et al ${ }^{[27]}$ & 5.7 years \\
\hline Andrea Ferretti et al ${ }^{[24]}$ & 6 years \\
\hline Georgios Koutras, et al ${ }^{[33]}$ & 6 months \\
\hline Chen Y, Zhu W, Wang W ${ }^{[34]}$ & 1 year and eight months \\
\hline
\end{tabular}

Present study assessed the short-term clinical outcome with median follow up time of 7.5 months. Although, some studies show that 6 months is adequate time to return to sporting activities after an ACL 
reconstruction ${ }^{[33]}$ and measurement of clinical scores and functional tests are valid at 6 month time, ${ }^{(31)}$ this short term follow up period is one of the limitations to this study.

\section{Conclusion}

Every patient with an ACL injury is different. ACL reconstruction techniques have advanced, but the principles remain the same and adhering to the 'anatomic' philosophy may allow better knee stability or enhance graft biology. This clinical study shows that arthroscopic ACL reconstruction using quadrupled semitendinosus revealed good subjective and objective results at a minimum follow-up time of 6 months. The results from this study confirmed the hypothesis that short-term clinical outcome are correlated with restoration and conservation of stability after isolated ACL rupture.

Hence, the present prospective study concludes that the anteromedial portal technique and anatomic placement of grafts is an effective surgical technique for treating ACL deficient knees. It restores stability with excellent clinical and functional results. Also single leg hop test is a reliable and valid functional test for evaluating post-operative outcome after an ACL reconstruction with the current available literature supporting this statement.

ACL: Anterior Cruciate Ligament

Abbreviations:

TT: Transtibial

AM: Anteromedial

VAS: Visual Analogue Scale

OPD: Out Patient Department

SPSS: Statistical Package for the Social Sciences

\section{References}

[1]. Bollen S . Ligament injuries of the knee-limping forward? Br J Sports Med. 1998 Mar;32(1):82-4.

[2]. Mohammadi F, Salavati M, Akhbari B, Mazaheri M, Mohsen Mir S, Etemadi Y. Comparison of functional outcome measures after ACL reconstruction in competitive soccer players: a randomized trial. J Bone Joint Surg Am. 2013;95:1271-7.

[3]. Andersson D, Samuelsson K, Karlsson J. Treatment of anterior cruciate ligament injuries with special reference to surgical technique and rehabilitation: an assessment of randomized controlled trials. Arthroscopy. 2009;25(6):653-685

[4]. Corry IS, Webb JM, Clingeleffer a J, Pinczewski L a. Arthroscopic reconstruction of the anterior cruciate ligament. A comparison of patellar tendon autograft and four-strand hamstring tendon autograft. Am J Sports Med 1999;27(3):444-54.

[5]. Ibrahim SA-R, Al-Kussary IM, Al-Misfer ARK, Al-Mutairi HQ, Ghafar SA, El Noor TA. Clinical evaluation of arthroscopically assisted anterior cruciate ligament reconstruction: patellar tendon versus gracilis and semitendinosus autograft. Arthroscopy. 2005;21(4):412-7.

[6]. Mihelic R, Jurdana H, Jotanovic Z, Madjarevic T, Tudor A. Long-term results of anterior cruciate ligament reconstruction: a comparison with non-operative treatment with a follow-up of 17-20 years. Int Orthop. 2011;35(7):1093-1097.

[7]. Chen L, Cooley V, Rosenberg T. ACL reconstruction with hamstring tendon. Orthop Clin North Am. 2003;34:9-18.

[8]. Gavriilidis I, Motsis EK, Pakos EE, Georgoulis AD, Mitsionis G, Xenakis TA. Transtibial versus anteromedial portal of the femoral tunnel in ACL reconstruction: A cadaveric study. Knee. 2008;15(5):364-7.

[9]. Bedi A, Musahl V, Steuber V, Kendoff D, Choi D, Allen AA, et al. Transtibial versus anteromedial portal reaming in anterior cruciate ligament reconstruction: An anatomic and biomechanical evaluation of surgical technique. Arthrosc - J Arthrosc Relat Surg Elsevier Inc.; 2011;27(3):380-90.

[10]. Aglietti P, Giron F, Losco M, Cuomo P, Ciardullo A, Mondanelli N Comparison between single-and double-bundle anterior cruciate ligament reconstruction: a prospective, randomized, single-blinded clinical trial. Am J Sports Med, 2010, 38:25-34

[11]. Jarvela T Double-bundle versus single-bundle anterior cruciate ligament reconstruction: a prospective, randomize clinical study. Knee Surg Sports Traumatol Arthrosc, 2007, 15:500-507

[12]. Ahlden M, Sernert N, Karlsson J, Kartus J. A Prospective Randomized Study Comparing Double- and Single-Bundle Techniques for Anterior Cruciate Ligament Reconstruction. Am J Sports Med 2013;41:2484-91.

[13]. Middleton KK, Hamilton T, Irrgang JJ, Karlsson J, Harner CD, Fu FH. Anatomic anterior cruciate ligament (ACL) reconstruction: a global perspective. Part 1. Knee Surg Sports Traumatol Arthrosc. 2014;22:1467-82.

[14]. Kocher MS, Steadman JR, Briggs K, Zurakowski D, Sterett WI, Hawkins RJ. Determinants of patient satisfaction with outcome after anterior cruciate ligament reconstruction. J Bone Jt Surg 2002;84-A:1560-72.

[15]. Neeb TB, Aufdemkampe G,Wagener JH, Mastenbroek L. Assessing anterior cruciate ligament injuries: the association and differential value of questionnaires, clinical tests, and functional tests. J Orthop Sports Phys Ther 1997;26(6):324-31

[16]. Lysholm J, Gillquist J. Evaluation of knee ligament surgery results with special emphasis on use of a scoring scale. Am J Sports Med. 1982; 10:150-4.

[17]. Briggs KK, Lysholm J, Tegner Y, Rodkey WG, Kocher MS, Steadman JR. The reliability, validity, and responsiveness of the Lysholm score and Tegner activity scale for anterior cruciate ligament injuries of the knee: 25 years later. Am J Sports Med. 2009;37:890-7

[18]. P S, Dominic D. Arthroscopic Acl Reconstruction Using Hamstring Tendon Autograft Fixed With Endobutton Cl ${ }^{\circledR}$ and Biorci - Ha (B) Interference Screw. J Evol Med Dent Sci. 2015;4(47):8190-9.

[19]. Tahmasebi M. N., Shahrezaee M., M.H. K, A. M. Reconstruction of Anterior Cruciate Ligament rupture: Results of 96 operations. Tehran Univ Med J. 2009;67:76-82.

[20]. Buchner M, Schmeer T, Schmitt H. Anterior cruciate ligament reconstruction with quadrupled semitendinosus tendon - minimum 6 year clinical and radiological follow-up. Knee. 2007;14:321-7. 
[21]. Harner CD, Honkamp NJ, Ranawat AS. Anteromedial Portal Technique for Creating the Anterior Cruciate Ligament Femoral Tunnel. Arthrosc J Arthrosc Relat Surg 2008;24(1):113-5.

[22]. Steiner M. Anatomic single-bundle ACL reconstruction. Sports Med Arthrosc. 2009;17(4):247-51

[23]. Alentorn-Geli E, Samitier G, Álvarez P, Steinbacher G, Cugat R. Anteromedial portal versus transtibial drilling techniques in ACL reconstruction: A blinded cross-sectional study at two- to five-year follow-up. Int Orthop. 2010;34(5):747-54.

[24]. Ferretti A, Monaco E, Giannetti S, Caperna L, Luzon D, Conteduca F. A medium to long-term follow-up of ACL reconstruction using double gracilis and semitendinosus grafts. Knee Surg Sports Traumatol Arthrosc 2011;19:473-8.

[25]. Bourke HE, Gordon DJ, Salmon LJ, Waller A, Linklater J, Pinczewski LA. The outcome at 15 years of endoscopic anterior cruciate ligament reconstruction using hamstring tendon autograft for "isolated" anterior cruciate ligament rupture. J Bone Jt Surg Br. 2012;94:630-7.

[26]. Keays SL, Bullock-Saxton JE, Keays a. C, Newcombe P a., Bullock MI. A 6-Year Follow-up of the Effect of Graft Site on Strength, Stability, Range of Motion, Function, and Joint Degeneration After Anterior Cruciate Ligament Reconstruction: Patellar Tendon Versus Semitendinosus and Gracilis Tendon Graft. Am J Sports Med. 2007;35:729-39

[27]. Cooley VJ, Deffner KT, Rosenberg TD. Quadrupled semitendinosus anterior cruciate ligament reconstruction: 5-year results in patients without meniscus loss. Arthrosc J Arthrosc Relat Surg 2001;17(8):a0170795.

[28]. Barber-Westin, S. D., Noyes, F. R., \& McCloskey, J. W. Rigorous statistical reliability, validity, and responsiveness testing of the Cincinnati knee rating system in 350 subjects with uninjured, injured or anterior cruciate ligament-reconstructed knees. The American Journal of Sports Medicine, 1999, 27(4), 402-416.

[29]. Greenberger, H. B.,\& Paterno, M. V. Relationship of knee extensor strength and hopping test performance in the assessment of lower extremity function. The Journal of Orthopaedic and Sports Physical Therapy, 1995, 22(5), 202-206.

[30]. Paterno, M. V.,\&Greenberger, H. B. The test-retest reliability of a one legged hop for distance in young adults with and without ACL reconstruction. Isokinetics and Exercise Science, 1996, 6, 1-6.

[31]. Shaw T, Chipchase LS, Williams MT. A users guide to outcome measurement following ACL reconstruction. Phys Ther Sport. 2004;5:57-67.

[32]. Mardani-Kivi M, Madadi F, Keyhani S, Karimi-Mobarake M, Hashemi-Motlagh K, Saheb-Ekhtiari K. Antero-medial portal vs. transtibial techniques for drilling femoral tunnel in ACL reconstruction using 4-strand hamstring tendon: a cross-sectional study with 1-year follow-up. Med Sci Monit 2012;18(11):CR674-9.

[33]. Koutras G, Papadopoulos P, Terzidis IP, Gigis I, Pappas E. Short-term functional and clinical outcomes after ACL reconstruction with hamstrings autograft: Transtibial versus anteromedial portal technique. Knee Surgery, Sport Traumatol Arthrosc. 2013;21(8):1904-9.

[34]. Chen Y, Zhu W, Wang W. Arthroscopic reconstruction of anterior cruciate ligament of knee by using autologous multi-stranded semitendinous tendon. Chinese journal of reparative and reconstructive surgery. 2007; 21:15-8. 